\title{
Determining Requirements for an Optimal Ad-Hoc Multicast Protocol in Mobile Health Care Training Systems
}

\author{
Anis Zarrad, Ahmed Redha Mahlous \\ Department of Computer Science and Information Systems, Prince Sultan University, Riyadh, Saudi Arabia \\ Email: azarrad@psu.edu.sa, armahlous@psu.edu.sa
}

Received 5 December 2014; accepted 3 April 2015; published 9 April 2015

Copyright (C) 2015 by authors and Scientific Research Publishing Inc.

This work is licensed under the Creative Commons Attribution International License (CC BY). http://creativecommons.org/licenses/by/4.0/

(c) (i) Open Access

\section{Abstract}

Nowadays, Health Care Training-based System (HCTS) is a vital component in the education and training of health care in 3D Virtual Environment (VE). The practice of HCTS continues to grow at rapid pace throughout all of the healthcare disciplines, however research in this field is still in its early stage. Increasingly, decision makers and developers look forward to offer more sophisticated, much larger, and more complex HCTS to serve the desired outcome and improve the quality and safety of patient care. Due to the rapidly increasing usage of personal mobile devices and the need of executing HCTS applications in environments that have no previous network infrastructure available, Mobile Health Care Training-based System (MHCTS) is an expected future trend. In such systems, medical staff will share and collaborate in a 3D virtual environment through their mobile devices in an ad-hoc network (MANET) in order to accomplish specific missions' typically surgical emergency room. Users are organized into various groups (Radiologists, Maternity departments, and General surgery etc...), and need to be managed by a multicast scheme to save network bandwidth and offer immersive sense. MHCTS is sensitive to networking issues, since interactive 3D graphics requires additional load due to the use of mobile devices. Therefore, we need to emphasize on the importance and the improvement of multicast techniques for the effectiveness of MHCTS and the management of collaborative group interaction. Research so far has devoted little attention to the network communication protocols design of such systems which is crucial to preserve the sense of immersion for participating users. In this paper, we investigate the effect of multicast routing protocol in advancing the field of Health care Training-based System to the benefit of patient's safety, and health care professional. Also, we address the issue of selecting a multicast protocol to provide the best performance for a particular e-health system at any time. Previous work has demonstrated that multicast operates at least as efficiently as traditional MAODV. A comprehensive analysis about various ad-hoc multicast routing protocols is proposed. The selection key factors for the right protocol for MHCTS applications were safety and robustness. To the best of our knowledge, this work will be the first initiative involving systematic 
literature reviews to identify a research gate for the use of multicast protocol in health care simulation learning community.

\title{
Keywords
}

\author{
Ad-Hoc, Multicast Protocol, Health Care Training System, 3D Virtual Environment, E-Health
}

\section{Introduction}

Mobile Health Care Training-based System (MHCTS) using virtual reality environment and telecommunication technologies is becoming important to both economic and patient's life safety. MHCS could be defined as a system that allows multiple trainees distributed geographically, to practice proper medical decision in the virtual environment, using their mobile devices in order to interact and collaborate with virtual patients. Such system let the trainee touch, feel, and manipulate virtual patients in safe way. Each trainee is represented by a graphical embodiment called avatar [1]. For the purpose of health care professional, the possibility to access detailed 3D virtual environment (VE) provides a promising alternative to traditional training. The unguided wireless medium and surrounding physical environment significantly disturb the radio transmissions, resulting in relatively unreliable communication channels. As a result, providing mobile healthcare VE simulator where conventional infrastructure-based communication facilities are not available, poses great challenges as more attention to network communication performance and 3D data exchange. Signal strength can easy degrades with distance between nodes. Therefore, there is a need to control network performance and consider the limited storage capabilities. Also, a specific problem related to the storage and performance capacity may occur, since users use their mobile devices with different characteristics to participate in MHCTS.

Currently, most of the health care simulators [2]-[4] are developed with desktop idea in mind and wired networks, thus hardware capabilities and network communication are not an issue. Health domain is largely benefit from collaborative virtual environment and wired network to provide the possibility of practicing medical procedures and share experiences with a remote tutor and other students [5]. In mobile health care virtual environment it is important to feed participants' devices only with necessary 3D data to display the user's viewpoint based on specific time and location in the VE. For example only avatars in the surgery room need to view the details about the room content. Avatars walking in the hospital corridor should not see what is inside the room. Nurses in the reception desks interact only with avatars in their area and can access their online data. Also, privacy is a central concern when dealing with capturing technologies for patients and can easily discomfort them.

To the researchers' knowledge, no attempt, except for work by Boukerch et al. [6], has been made to apply multicast protocol in e-health. Several steps have been made toward 3D rendering, streaming and 3D graphical display. However, network communication performance when dealing with VE over Ad-hoc networks has never been considered as an important concern. Emergency response efforts in critical situation are a must. For example in a car crash accident, wounded people are taken to the local hospital where they are forwarded directly to ER room. Figure 1 shows an ER scenario in a hospital reception area.

An initial assessment and management of critically injured people require a rapid intervention with the following actions:

- Preparation of a resuscitation area and necessary monitoring equipments.

- Immediate call of medical staff.

- Prompt laboratory and radiology backup.

Once patients are received, immediately medical staff can start simulations to control and manage the ER wounded situation. All medical staff (nurses, doctors, Radiologists etc...) need to join the VE and collaborate together to deal with this situation. Surgeon need to manipulate virtual tissues and 3D anatomical models of organs through the same surgical tool usually used in real situation, while viewing images interactions on his mobile devices. 3D data and other shared data should be exchanged between participants in the VE.

MHCTS is a network delay- and bandwidth-sensitive application, especially in terms of message updates that reflect users' actions in the Virtual Environment (VE). Any interaction with the shared environment should be received in an acceptable delay to maintain the feeling of immersion and guarantee reality. In typical applications, 


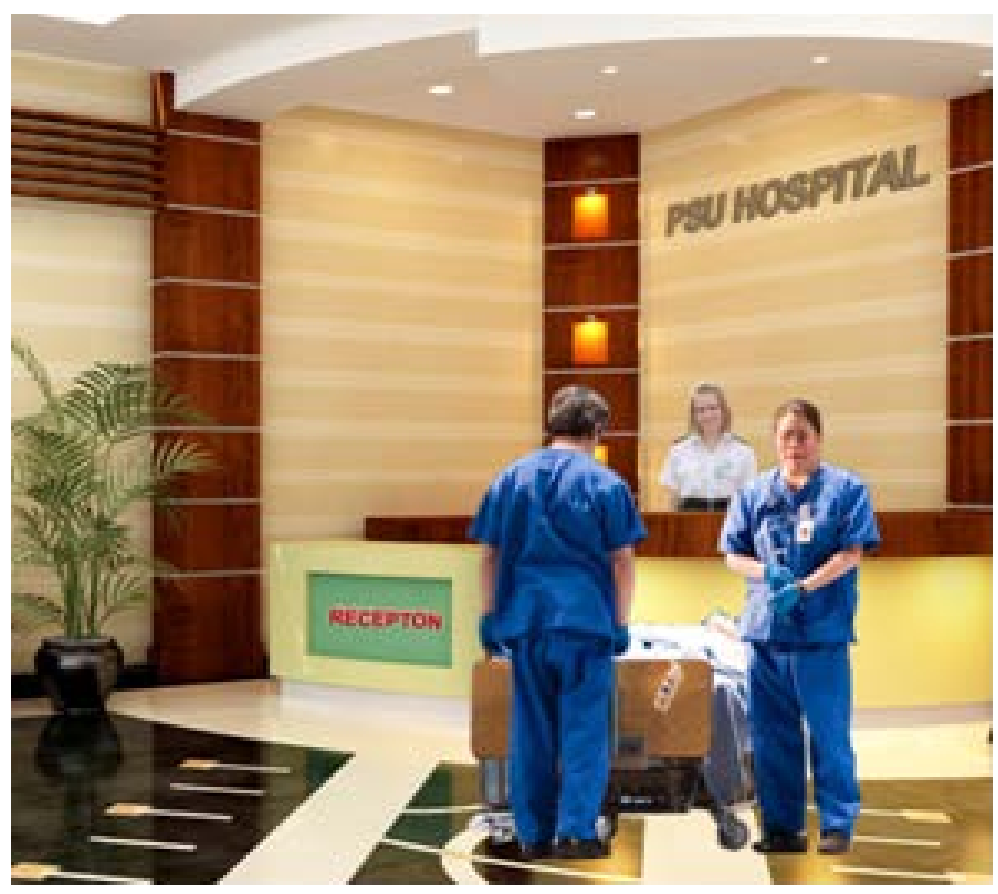

Figure 1. Emergency scenario.

network latency tolerance [7] usually varies between $10 \mathrm{~ms}$ to $1000 \mathrm{~ms}$. However, it is argued in [8] that network latency in VE applications should not exceed $100 \mathrm{~ms}$ to preserve the immersion sense, but may reach a maximum of $200 \mathrm{~ms}$ [9] in some cases.

Multicast can be a primitive solution to cope with network delay and messages filtering. Only interested people will receive specific data. There is no need for nurses Avatars to receive data about the surgical procedure; only surgeons Avatars will receive the required data. Designing a multicast protocol to handle the network requirements and the sense of immersive for Mobile Health care Training-based (MHCTS) systems is a challenging task and requires expertise in systems engineering, robotics engineering, computer science, and medicine. In our previous work [6] we developed a multicast protocol based on Gnutella peer-to-peer networks to support mobile virtual environment. In this paper we work on providing a literature reviews for potential Multicast protocols in ad-hoc networks that can be applied in Mobile medical health care simulation. Our measurements and analysis of multicast protocols are driven by three primary concerns. The first one is the sensitivity and satisfaction of robustness because MHCTS should work under difficult circumstances (avoiding single points of failure). Robustness is an indicator for the resilience to errors and misinformation such as error prevention, fault detection, fault tolerance, redundancy, recovery and restart blocks. The second one is handling mobility and keeping nodes connected. The last concern has to deal with the decision of whether to propose a new protocol to meet MHCTS objectives or the use of an existing one.

The remainder of the paper is organized into three main sections. Section 2 provides a general idea about some used Multicast routing protocols over Ah-hoc network. Section 3 presents existing Multicast protocols applied in medicine. Section 4 concludes and presents future direction for the development of the new protocol.

\section{Related Work a Short Survey}

In recent years, a number of multicast protocols for ad hoc networks have been proposed. Most multicast communications in the internet involves the use of routing trees. The fundamental approach consists of creating a routing tree for a group of nodes (routers) in order to communicate efficiently. Thus, packets or message sent to all the routers in the tree traverses each router and link in the tree only once. Creating and using multicast routing trees have been extensively studied in Mobile ad hoc network and Wireless Sensor network (WSN). In order to meet the MHVTS real time requirement we classify proposed multicast protocols based on their robustness satisfaction, and multicast efficiency in order . 
Multicast Operation of the Ad-hoc On-Demand Distance Vector Routing Protocol [10] MAODV is perhaps the most well know multicast protocol that represent a shared-tree-based protocol that is extended from AODV [11]. In MAODV, all members of a multicast group are formed into a tree, and the root of the tree is the group leader (first node join the tree). Multicast data packets are propagated among the tree. With the unicast route information of AODV, MAODV constructs the shared tree more efficiently and has low control overhead. Due to node mobility a tree partition may happen. A group member $\mathrm{Q}$ whose group leader has a lower IP address than any other group leader will inform its group leader to stop the leader's role. Node Q then sends a message to ask the group leader with the highest IP address to be the new group leader of the final merged tree. Advantages: With the unicast route information, the multicast tree can be constructed more quickly and efficiently. Disadvantages: The group leader continues flooding Group Hello messages even if no sender for the group exists.

Authors in [12] summarize and comparatively analyze the routing mechanisms of various existing multicast routing protocols according to the characteristics of mobile Ad Hoc network. From those protocols we find Ad Hoc Multicast Routing Protocol Utilizing Increasing Id-numbers (AMRIS) [13]. It is an on-demand shared-treebased protocol which dynamically assigns every node in a multicast session an id-number known as msm-id. The msm-id provides each node with an indication of its "logical height" in the multicast delivery tree. Excluding the root node, each other node should have one parent that has a logical height (msm-id) that is smaller than it. A special node that is known as Sid with the smallest msm-id initiates initialization phase (multicast session) by broadcasting a NEW-SESSION message, in which each participant receiving the NEW-SESSION calculates its initial msm-id dynamically based on the value found in the message. If nodes receive multiple NEWSESSION messages (for the same multicast session), they will only chose one message to process. Thus, the NEW-SESSION message thus travels in an expanding ring fashion outwards from Sid. In the initial phase, nodes closer to Sid will generally have smaller msm-ids than those further away.

These id-numbers help nodes know which neighbors are closer to the Sid and this reduces the cost to repair link failures (e.g. due to mobility terrain) and re-join the delivery tree in a localized fashion without causing permanent routing loops. One of the key features of AMRIS is on the way it provides routing information to other nodes. It doesn't use a unicast routing protocol, AMRIS maintains a Neighbor-Status table which stores the list of existing neighbors and their msm-ids.

The msm-ids allow nodes that have broken off from the delivery tree (e.g. due to mobility, terrain) to re-join the delivery tree in a localized fashion without causing permanent routing loops. One of the key features of AMRIS is that it does not depend on the unicast routing protocol to provide routing information to other nodes. AMRIS maintains a Neighbor-Status table which stores the list of existing neighbors and their msm-ids. Each node sends a periodic beacon to signal their presence to neighboring nodes. The beacon contains the msm-ids that each node presently has. However some disadvantages are present in AMRIS such as joining and re-joining of a node may take long time and waste much bandwidth since each node tries potential parent nodes arbitrarily and the usage of periodic beacons consumes bandwidth. Other protocol analyzed by [12], is the Lightweight Adaptive Multicast protocol (LAM) [14]. It provides services by using of the CBT (Core Based Tree) Algorithm and the TORA (Temporally-Ordered routing Algorithm). Similar with CBT, for each multicast group, LAM constructs a shared multicast tree whose center is the core node.

Two variables are maintained by nodes in LAM: POTENTIAL-PARENT and PARENT, and two lists, POTENTIALCHILD-LIST and CHILD-LIST. The PARENT variable is used to remember the parent node in the multicast tree, while the CHILD-LIST stores identities of one-hop child nodes in the multicasting tree. The POTENTIAL data structure will be used when the nodes are in a "join" or "rejoin" state. In mobile Ad Hoc network environment LAM has bad robustness. Another multicast WSN protocol can be found in literature. In [15] authors started by presenting a classification of the most common WSN multicast procedures depending on the way a target group is identified by the means of geographic position. They proposed a new protocol named Dijkstra-based Localized Energy-Efficient Multicast Algorithm (DLEMA) which is based on Localized EnergyEfficient Multicast Algorithm (LEMA) [16]. The first phase consist on building Minimum spanning Tree based (MST) on Kruskal's algorithm [17] seen from perspective of ssource-a node currently routing a message. A member of a multicast group is added to MST only if at least one neighbor of $s$ provides Euclidean advance towards that destination, or a perimeter relay node can be found.

For all destinations meeting the criteria, the current node becomes the root of the tree with edge costs reflecting the distance between nodes. Sensors constituting multicast group are the leaves or intermediate nodes. The only additional information about network topology available to $s$ is the location of the nodes operating within 
its radio range. Therefore, the current node selects relay neighbors that are in the closest possible geographical proximity of each subset of destinations. In the second phase, LEMA uses Dijkstra's algorithm [18] to determine Energy Shortest Paths (ESPs) leading to neighbors that provide maximum geographical advance towards desired destinations. The procedure is based on the observation that ESP may consist of nodes not providing Euclidean advance but still minimizing total energy required to transport message from $s$ to the end of the path. LEMA uses a localized source routing technique. A path determined in current node is stored in Source Routing Header (SRH) added to the message, while each sensor receiving a message with SRH should follow the requested route. Therefore, nodes that do not provide direct geographical advance can be designated as relays on the Energy Shortest Path to increase energy conservation of the network.

Unlike LEMA, DLEMA uses Dijkstra's algorithm to calculate shortest path tree (SPT) in its first phase. A performance evaluation of DLEMA, LEMA and Cone-based Forwarding Area Multicast tree (CoFAM) [19] was conducted through an extensive simulation. The results showed the outperformance of DLEMA over the other two protocols. In [20] authors present a receiver-initiated protocol called Core Assisted Mesh Protocol (CAMP). The multicast routing consists of building a shared mesh for each multicast group. It assumes that an underlying unicast routing protocol provides correct distances to known destinations. CAMP ensures that the shortest paths from receivers to sources (called reverse shortest paths) are part of a group's mesh. The number of packets coming from the reverse path for a sender indicates whether the node is on the shortest path. CAMP extends the basic receiver-initiated approach introduced in the core-based tree (CBT) protocol [21] for the creation of multicast trees to enable the creation of multicast meshes. CAMP advantages are: First, It constructs a mesh without control packet flooding. Second Node joining and shortest path procedure incurs very low overhead. However in the other hand CAMP has to rely on certain unicast routing protocols and offer high storage overhead.

\section{Preliminaries: Multicast Routing Protocols Requirements for Mobile Health Care Training-Based System}

In Mobile Health Care Training-based System users can interact and collaborate together. Users are assembled into groups based on their interest in the virtual environment. Users' immersive sensation is a critical issue that must be considered when designing such applications to offer certain realism. Therefore, MHCTS applications are considered to be sensitive to networking issues. During a higher transmission rates due to the use additional loads of 3D graphical data, it is hard to maintain real-time requirements and offer better performance. In general a multicast group is composed of senders and receivers. For connecting senders and receivers, each protocol constructs either a tree or a mesh as the routing structure. There are some nodes called forwarding nodes in the routing structure that are not interested in multicast packets but act as routers to forward them to receivers. Group members (senders and receivers) and forwarding nodes are also called tree or mesh nodes depending on the routing structure. In the routing structure, a node is an upstream (downstream) node of another node if it is closer to (farther away) the root of the tree. If the two nodes belong to the same link, the upstream (downstream) node is also called the parent (child) of the other node.

\section{a) E-Health Requirements}

E-Health systems have been well researched and studied for many numbers of years [22]. Recent advancements in mobile technologies have put a mobility requirement on traditional eHealth requirements. Mobility does offer more magnitude to the system, specifically the ability to work autonomously in a mobile environment without losing interest to the application. Classical eHealth requirements are: Efficient communication mechanism is crucial to reduce the network traffic generated by users' interaction messages, so that large scalability can be supported without affecting the overall performance of the proposed system without losing interest in the application. Adaptability should be also considered when developing multicast protocol, users may participate with different mobile devices having different criteria and capabilities such as, hardware, operating system, storage system, network capacity etc..., and therefore resulted protocol must be well adapted to different devices. In mobile environments, adaptability is considered a fundamental requirement due to the limitations of certain mobile devices.

In such system medical staffs collaborate toward specific objective. The state of the virtual environment and 3D data must be maintained during the whole mission and carried out even when user owner leave the mission. Persistency is important especially in eHealth system, for example when a nurse gives a medicine to a patient, 
the medical report should be updated. Thus, when the nurse finishes her shift and leaves the virtual environment, new comer is informed. Persistency can be achieved in multicast protocol by assigning backup nodes in the network to store all relevant actions and data for the mission. The last requirement we believe it important is the Operability, the system use standards and patterns in order to allow interactions between heterogeneous systems.

Attempting to build e-Health systems with all of the aforementioned requirements require immense efforts. In this work, we diminish the boundary requirements so as to consider only the mobility, and efficient network communication. The connectivity of the mobile nodes, route setup and repair time are the major factors that affect network performance.

\section{b) Unicast versus Multicast versus Broadcast Routing}

In the literature existing routing techniques can be classified either as Multicast, broadcast and unicast. Unicast is defined as a packet needs to go from a single source to a single destination. The communication is from a single node to another single node. There is one device transmitting a message destined for one receives. Contrary in multicast routing the packet needs to go from a single source to several destinations in a given address range not defined by any standard IP address and mask combination. This broadcast could reach all hosts on the subnet, all subnets, or all hosts on all subnets. Current routers, during the broadcast communication block IP broadcast traffic and restrict only to the local subnet. Multicast communication uses a distinct set of addresses. In broadcast routing the packet needs to go from a single source to every receiver exist in the network. The scope of the broadcast is limited to a broadcast domain. Cleary Multicast protocol can be an alternative solution to handle the e-Health application, because major critical activities are classified by department, and groups. Example surgery group, emergency departments etc...Also network bandwidth usability is more efficient because of multiple streams. Therefore, routing productivity is increased.

In [2] authors described the importance of network issue as a multicast protocol when using medical simulation in peer-to-peer network to achieve better realism during simulations and improve the immersive sense for all users participating in collaborative virtual environment. The proposed protocol is implemented in the collaborative module of the CyberMed VR framework. Greenhalgh et al. [3] describe a multicast protocol for Large Scale Collaborative Virtual Environments where the source node transmits only one packet information to a specific group of interested receivers. Instead of sending $\mathrm{N}$ separate but identical packets to each of $\mathrm{N}$ receivers, one multicast packet can be sent and multicast guarantees it will reach all $\mathrm{N}$ receivers. Therefore better scalability and decrease the use of the network bandwidth on CVEs, which allow better Quality and experience of the VE. Boukerch et al. [4] proposed an alternative solution of collaborative, haptic, audio and visual environments (C-HAVE) in order to cope with network delay, scalability, reliability and synchronization problem when the users are geographically distributed. A hybrid solution that incorporate four main protocols (the synchronous collaboration transport protocol (SCTP), the selective reliable transmission protocol (SRTP), the reliable multicast transport protocol (RMTP) and the scalable reliable multicast (SRM)) is proposed for Brain Tumor Tele-Surgery application.

\section{Discussion}

So far, the mobile ad-hoc research community group has proposed many multicast routing protocols, each one with its advantages and disadvantages in terms of robustness and efficiency to adapt to different network environment. For instance, the advantage of AMRIS protocol is that the nodes do not need to store any global information as it has a locality in link repair. By virtue of multicast updating cycle where each node broadcasts beacon messages containing its ID and other information, therefore, in case of a link failure, node with a bigger ID will rejoins the multicast tree rapidly. However, in case of rapid node moving and in term of robustness, AMRIS sees its performance decline, this is due to the fact that, it is classified as a tree-based multicast routing that doesn't have a redundant path between two nodes. Another drawback is when the node density increases, AMRIS needs to periodically send Beacon message to maintain the multicast tree, which will be greatly conflicted when the node's density is comparatively big. Despite AMRIS is relatively lightweight in terms of state, it is not in term of bandwidth, since control messages are sent periodically rather than being data-driven.

LAM is based on the CBT approach to building the multicast delivery tree, with one CORE to a group and provides multicasting service for large scale mobile ad hoc networks; LAM is not very robust, especially in a MANET environment. DLEMA is considered as a successful enhancement of LEMA especially in larger and denser WSN. It has the advantage of providing low delay and high success rate. However it is less robust in an environment which has a continuous link failure. 


\section{Conclusion}

Mobile Health Care Training-based System (MHCTS) is still a relatively new field, where it can play an important role in the analysis of the collaboration between users, technology, and the healthcare domain. Such system involves the need of analysis to identify learning outcomes and test possible scenarios in 3D graphical environment before being applied to real life. MHCTS is a complex system that operates in complex networked environment to offer an immersive sense to all users in the VE. The goal of this paper was to identify the importance of multicast protocol in serving realistic aspect while they perceive user's actions distributed geographically. Another goal was to analyze some existing multicast protocols over ad-hoc network to identify the future direction of developing multicast protocol for Mobile Health Care Training-based System (MHCTS). Robustness and multicast efficiency are important factors to be considered in such application. If the degree of robustness is low, the packet delivery ratio will drop and high control overhead will be incurred. Also some other protocols implement the shortest path algorithm paths between senders and receivers. Thus, link failures and network latency are reduced. In this direction, as a future work, we will develop a new protocol that will take into consideration multicast efficiency and robustness in order to fill a gap in the E-health field.

\section{Acknowledgements}

Authors would like to express their thanks to Prince Salman Research and Translation Center (PSRTC) in Prince Sultan University.

\section{References}

[1] Peterson, M. (2005) Learning Interaction in an Avatar-Based Virtual Environment: A Preliminary Study. Journal Pacific Association for Computer Assisted Language Learning, 1, 29-40.

[2] Paiva, P.V.F., Machado, L.S. and de Oliveira, J.C. (2012) A Peer-to-Peer Multicast Architecture for Supporting Collaborative Virtual Environments (CVEs) in Medicine. Proceedings of 14th Symposium on Virtual and Augmented Reality, Rio Janiero, 28-31 May 2012, 165-173.

[3] Greenhalgh, C. and Benford, S. (1997) A Multicast Network Architecture for Large Scale Collaborative Virtual Environments. ECMAST*97, Milan, May 1997, 21-23.

[4] Boukerche, A., Maamar, H. and Hossain, A. (2007) A Performance Evaluation of a Hybrid Multicast Transport Protocol for a Distributed Collaborative Virtual Simulation of a Brain Tumor Tele-Surgery Class of Applications. 12th IEEE Symposium on Computers and Communications, ISCC 2007, Aveiro, 1-4 July 2007, 975-980.

[5] Paiva, P.V.F., Machado, L.S. and Oliveira, J.C. (2012) An Experimental Study on CHVE's Performance Evaluation. Studies in Health Technology and Informatics, 173, 328-330.

[6] Boukerche, A. and Ren, Y. (2009) A Secure Mobile Healthcare System Using Trust-Based Multicast Scheme. IEEE Journal on Selected Areas in Communications, 27, 387-399.

[7] Claypool, M. and Claypool, K. (2006) Latency and Player Actions in Online Games. Communications of the ACM, Special Issue: Entertainment Networking, 49, 40-45.

[8] Wloka, M. (1995) Lag in Multiprocessor VR. Presence: Teleoperators and Virtual Environments (MIT Press), 4, 5063.

[9] Park, K. and Kenyon, V. (1999) Effects of Network Characteristics on Human Performance in a Collaborative Virtual Environment. Proceedings of the IEEE Virtual Reality, Houston, 13-17 March 1999, 104-111.

[10] Perkins, C.E. and Royer, E.M. (1999) Ad-Hoc On-Demand Distance Vector Routing. Proceedings WMCSA'99. Second IEEE Workshop on Mobile Computing Systems and Applications, 90-100. http://dx.doi.org/10.1109/MCSA.1999.749281

[11] Royer, E.M. and Perkins, C.E. (1999) Multicast Operation of the Ad-Hoc On-Demand Distance Vector Routing Protocol. In: Proceedings of the 5th Annual ACM/IEEE International Conference on Mobile Computing and Networking, ACM, New York, 207-218. http://dx.doi.org/10.1145/313451.313538

[12] Xiang, M. (2012) Analysis on Multicast Routing Protocols for Mobile Ad Hoc Networks. Proceedings of the International Conference on Solid State Devices and Materials Science, Macao, 1-2 April 2012, 1787-1793.

[13] Wu, C.W., Tay, Y.C. and Toh, C.K. (1998) Ad-Hoc Multicast Routing Protocol Utilizing Increasing Id-Numbers (AMRIS) Functional Specification. Internet Draft, November 1998.

[14] Garcia-Luna-Aceves, J.J. and Madruga, E.L. (1999) The Core-Assisted Mesh Protocol. IEEE Journal on Selected Areas in Communications, 17, 1380-1394. 
[15] Musznicki, B., Tomczak, M. and Zwierzykowski, P. (2012) Dijkstra-Based Localized Multicast Routing in Wireless Sensor Networks. Proceedings of the 8th International Symposium on Communication Systems Networks \& Digital Signal Processing (CSNDSP), Poznan, 18-20 July 2012, 1-6.

[16] Sanchez, J.A. and Ruiz, P.M. (2006) LEMA: Localized Energy-Efficient Multicast Algorithm Based on Geographic Routing. Proceedings of the 31st IEEE Conference on Local Computer Networks, Tampa, 14-16 November 2006, 3-12. http://dx.doi.org/10.1109/LCN.2006.322092

[17] Kruskal, J.B. (1956) On the Shortest Spanning Subtree of a Graph and the Traveling Salesman Problem. Proceedings of the American Mathematical Society, 7, 48-50. http://dx.doi.org/10.1090/S0002-9939-1956-0078686-7

[18] Dijkstra, E.W. (1959) A Note on Two Problems in Connexion with Graphs. Numerische Mathematik, 1, $269-271$. http://dx.doi.org/10.1007/BF01386390

[19] Zhang, W., Jia, X., Huangand, C. and Yang, Y. (2005) Energy-Aware Location Aided Multicast Routing in Sensor Networks. Proceedings of the International Conference on Wireless Communications, Networking and Mobile Computing, Wuhan, 23-26 September 2005, 901-904.

[20] Garcia-Luna-Aceves, J.J. and Madruga, E.L. (1999) The Core-Assisted Mesh Protocol. IEEE Journal on Selected Areas in Communications, 17, 1380-1394. http://dx.doi.org/10.1109/49.779921

[21] Ballardie, A., Francis, P. and Crowcroft, J. (1993) Core Based Trees (CBT): An Architecture for Scalable Inter-Domain Multicast Routing. Proceedings of the ACM SIGCOMM' 93 Conference on Communications Architectures, Protocols and Applications, San Francisco, September 13-17 1993, $13-17$.

[22] Boukerche, A., Zarrad, A. and Araujo, R. (2007) A Novel Gnutella Application Layer Multicast Protocol for Collaborative Virtual Environments over Mobile Ad-Hoc Networks. Proceedings of the IEEE Wireless Communications and Networking Conference, Kowloon, 11-15 March 2007, 2825-2830. 